\title{
Open manifolds with nonnegative Ricci curvature and large volume growth
}

\author{
Changyu Xia
}

\begin{abstract}
In this paper, we study complete open $n$-dimensional Riemannian manifolds with nonnegative Ricci curvature and large volume growth. We prove among other things that such a manifold is diffeomorphic to a Euclidean $n$-space $R^{n}$ if its sectional curvature is bounded from below and the volume growth of geodesic balls around some point is not too far from that of the balls in $R^{n}$.
\end{abstract}

Mathematics Subject Classification (1991). (1985 Revision): 53C20; Secondary 53C21, 53R70, 31C12.

Keywords. Open manifolds, nonnegative Ricci curvature, large volume growth.

\section{Introduction}

Let $(M, g)$ be an $n$-dimensional complete Riemannian manifold with nonnegative Ricci curvature. The relative volume comparison theorem [BC, GLP] says that the function $r \rightarrow \frac{\operatorname{vol}[B(p, r)]}{\omega_{n} r^{n}}$ is monotone decreasing, where $B(p, r)$ denotes the geodesic ball around $p \in M$ with radius $r$ and $\omega_{n}$ is the volume of the unit ball in the Euclidean space $R^{n}$. Define $\alpha_{M}$ by

$$
\alpha_{M}=\lim _{r \rightarrow \infty} \frac{\operatorname{vol}[B(p, r)]}{\omega_{n} r^{n}} .
$$

It is easy to show that $\alpha_{M}$ is independent of $p \in M$, hence it is a global geometric invariant of $M$. We always have

$$
\alpha_{M} \omega_{n} r^{n} \leq \operatorname{vol}[B(x, r)] \leq \omega_{n} r^{n}, \quad \forall r>0, \quad \forall x \in M .
$$

We say $(M, g)$ has large volume growth if $\alpha_{M}>0$. It should be noticed that, in this case, $0<\alpha_{M} \leq 1$ and when $\alpha_{M}=1, M$ is isometric to $R^{n}$ by Bishop-Gromov comparison theorem [BC, GLP].

A manifold $M$ is said to have finite topological type if there is a compact domain $\Omega$ whose boundary $\partial \Omega$ is a topological manifold such that $M \backslash \Omega$ is homeomorphic 
to $\partial \Omega \times[0, \infty)$. Abresch-Gromoll $[\mathrm{AG}]$ first obtain the finiteness of topological type for complete $n$-manifolds $(M, g)$ with $\operatorname{Ric}_{M} \geq 0$ and small diameter growth $\operatorname{diam}(p, r)=o\left(\frac{1}{r^{n}}\right)$, provided that the sectional curvature $K_{M} \geq K_{0}>-\infty$.

Let $(M, g)$ be an $n$-dimensional complete manifold with $\operatorname{Ric}_{M} \geq 0$ and $\alpha_{M}>0$. It has been proved by $\mathrm{Li}[\mathrm{L}]$ that $M$ has finite fundamental group. Anderson [A] has showed that the order of the fundamental group of $M$ is bounded from above by $\frac{1}{\alpha_{M}}$. Perelman $[\mathrm{P}]$ has proved that there is a small constant $\epsilon(n)>0$ depending only on $n$ such that if $\alpha_{M}>1-\epsilon(n)$, then $M$ is contractible. It has been shown by Shen [S2] that $M$ has finite topological type, provided that $\frac{\operatorname{vol}[B(p, r)]}{\omega_{n} r^{n}}=\alpha_{M}+o\left(\frac{1}{r^{n-1}}\right)$ and, either the conjugate radius $\operatorname{conj}_{M} \geq c>0$ or the sectional curvature $K_{M} \geq K_{0}>-\infty$. Petersen [Pe] conjectured that if $\alpha_{M}>\frac{1}{2}$ then $M$ is diffeomorphic to $R^{n}$. Recently, Cheeger and Colding [CC] gave a partial answer to Petersen's conjecture. In fact, they proved that there exists a small constant $\delta(n)>0$ such that if $\alpha_{M} \geq 1-\delta(n)$, then $M$ is diffeomorphic to $R^{n}$. Another result which supports stongly Petersen's conjecture has been obtained by do Carmo and the author recently in [CX].

In the present paper, we study complete manifolds with nonnegative Ricci curvature and large volume growth. Let $M$ be a complete manifold and $p \in M$ be fixed; we say that $K_{p}^{\mathrm{min}} \geq c$ if for any minimal geodesic $\gamma$ issuing from $p$ all sectional curvatures of the planes which are tangent to $\gamma$ are greater than or equal to $c$. This notion was first introduced by Klingenberg $[\mathrm{K}]$.

Theorem 1.1. Let $(M, g)$ be a complete Riemannian n-manifold with Ricci curvature $\operatorname{Ric}_{M} \geq 0, \alpha_{M}>0$. Suppose that $K_{p}^{\text {min }} \geq-C$ for some point $p \in M$ and some positive constant $C$. If for all $r>0$, we have

$$
\frac{\operatorname{vol}[B(p, r)]}{\omega_{n} r^{n}}<\left\{1+2^{-n}\left(\frac{1}{8 \sqrt{C} r} \log \left(\frac{2}{1+e^{-2 \sqrt{C} r}}\right)\right)^{n-1}\right\} \alpha_{M},
$$

then $M$ is diffeomorphic to $R^{n}$.

The following result is a generalization of Shen's theorem mentioned above.

Theorem 1.2. Let $(M, g)$ be a complete Riemannian n-manifold with Ricci curvature $\operatorname{Ric}_{M} \geq 0, \alpha_{M}>0$. Suppose that $K_{p}^{\min } \geq-C$ for some $p \in M$ and $C>0$. If

$$
\limsup _{r \rightarrow+\infty}\left\{\left(\frac{\operatorname{vol}[B(p, r)]}{\omega_{n} r^{n}}-\alpha_{M}\right) r^{n-1}\right\}<2^{-n}\left(\frac{\log 2}{8 \sqrt{C}}\right)^{n-1} \alpha_{M}
$$

then $M$ has finite topological type.

Let $(M, g)$ be an $n$-dimensional complete noncompact Riemannian manifold. Fix a point $p \in M$. For any $r>0$, let

$$
k_{p}(r):=\inf _{M \backslash B(p, r)} K
$$


where $B(p, r)$ is the open geodesic ball around $p$ with radius $r, K$ denotes the sectional curvature of $M$, and the infimum is taken over all the sections at all points on $M \backslash B(p, r)$. It is easy to see that $k_{p}(r) \leq 0$ and that $k_{p}(r)$ is a monotone function of $r$.

U. Abresch [A] proved that if $\int_{0}^{\infty} r k_{p}(r) d r>-\infty$, then $M$ is of finite topological type. Recently, Sha and Shen [SS] showed that a complete open Riemannian manifold $M$ has finite topological type if $\operatorname{Ric}_{M} \geq 0, \alpha_{M}>0$ and

$$
k_{p}(r) \geq-\frac{C}{1+r^{2}}
$$

for some constant $C>0$ and all $r>0$.

In this paper we then prove the

Theorem 1.3. Given $C>0$, and an integer $n \geq 2$, there is a positive constant $\epsilon=\epsilon(n, C)$ such that any complete Riemannian n-manifold $M$ with Ricci curvature $\operatorname{Ric}_{M} \geq 0, \alpha_{M}>0, k_{p}(r) \geq-\frac{C}{1+r^{2}}$ and

$$
\frac{\operatorname{vol}[B(p, r)]}{\omega_{n} r^{n}} \leq(1+\epsilon) \alpha_{M}
$$

for some $p \in M$ and all $r>0$ is diffeomorphic to $R^{n}$.

Now we list the following Toponogov-type comparison theorem for complete manifolds with $K_{p}^{\min } \geq c$ obtained by Machigashira which will be used in this paper. Let $M^{2}(c)$ be the complete simply connected surface of constant curvature $c$. Throughout this paper, all geodesics are assumed to have unit speed.

Lemma 1.1 ([M1], [M2]) Let $M$ be a complete Riemannian manifold and $p$ be a point of $M$ with $K_{p}^{\mathrm{min}} \geq c$.

(i) Let $\gamma_{i}:\left[0, l_{i}\right] \rightarrow M, i=0,1,2$ be minimal geodesics with $\gamma_{1}(0)=\gamma_{2}\left(l_{2}\right)=$ $p, \gamma_{0}(0)=\gamma_{1}\left(l_{1}\right)$ and $\gamma_{0}\left(l_{0}\right)=\gamma_{2}(0)$. Then, there exist minimal geodesics $\tilde{\gamma}_{i}$ : $\left[0, l_{i}\right] \rightarrow M^{2}(c), i=0,1,2$ with $\tilde{\gamma_{1}}(0)=\tilde{\gamma_{2}}\left(l_{2}\right), \tilde{\gamma_{0}}(0)=\tilde{\gamma_{1}}\left(l_{1}\right)$ and $\tilde{\gamma_{0}}\left(l_{0}\right)=\tilde{\gamma_{2}}(0)$ which are such that

$$
L\left(\gamma_{i}\right)=L\left(\tilde{\gamma}_{i}\right) \text { for } i=0,1,2
$$

and

$$
\begin{aligned}
& \angle\left(-\gamma_{1}^{\prime}\left(l_{1}\right), \gamma_{0}^{\prime}(0)\right) \geq \angle\left(-\tilde{\gamma}_{1}{ }^{\prime}\left(l_{1}\right), \tilde{\gamma}^{\prime}(0)\right), \\
& \angle\left(-\gamma_{0}^{\prime}\left(l_{0}\right), \gamma_{2}^{\prime}(0)\right) \geq \angle\left(-{\tilde{\gamma_{0}}}^{\prime}\left(l_{0}\right),{\tilde{\gamma_{2}}}^{\prime}(0)\right) .
\end{aligned}
$$

(ii) Let $\gamma_{i}:\left[0, l_{i}\right] \rightarrow M, i=1,2$ be two minimizing geodesics starting from p. Let $\tilde{\gamma}_{i}:\left[0, l_{i}\right] \rightarrow M^{2}(c)$ for $i=1,2$ be minimizing geodesics starting from same point such that $\angle\left(\gamma_{1}^{\prime}(0), \gamma_{2}^{\prime}(0)\right)=\angle\left({\tilde{\gamma_{1}}}^{\prime}(0),{\tilde{\gamma_{2}}}^{\prime}(0)\right)$. Then $d\left(\gamma_{1}\left(l_{1}\right), \gamma_{2}\left(l_{2}\right)\right) \leq$ $d_{c}\left(\tilde{\gamma}_{1}\left(l_{1}\right), \tilde{\gamma}_{2}\left(l_{2}\right)\right)$, where $d_{c}$ denotes the distance function in $M^{2}(c)$. 


\section{Proof of Theorem 1.1 and Theorem 1.2}

Let $M$ be an $n$-dimensional Riemannian manifold and $1 \leq k \leq n-1$. If for any point $x \in M$ and any $(k+1)$-mutually orthogonal unit tangent vectors $e, e_{1}, \ldots, e_{k} \in$ $T_{x} M$, we have $\sum_{i=1}^{k} K\left(e \wedge e_{i}\right) \geq 0$, we say that the $k$-th Ricci curvature of $M$ is nonnegative and denote this fact by $\operatorname{Ric}_{M}^{(k)} \geq 0$. Here, $K\left(e \wedge e_{i}\right)$ denote the sectional curvature of the plane spanned by $e$ and $e_{i}(1 \leq i \leq k)$. Notice that if $\operatorname{Ric}_{M}^{(k)} \geq 0$ then $\operatorname{Ric}_{M} \geq 0$.

We shall prove the following more general theorem than Theorem 1.1.

Theorem 2.1. Let $(M, g)$ be a complete Riemannian n-manifold with $\operatorname{Ric}_{M}^{(k)} \geq$ $0, \alpha_{M}>0$. Suppose that $K_{p}^{\text {min }} \geq-C$ for some $C>0$ and $p \in M$. If for all $r>0$, we have

$$
\frac{\operatorname{vol}[B(p, r)]}{\omega_{n} r^{n}}<\left\{1+2^{-n}\left(\frac{1}{8 \sqrt{C} r} \log \left(\frac{2}{1+e^{-2 \sqrt{C} r}}\right)\right)^{\frac{k n}{k+1}}\right\} \alpha_{M},
$$

then $M$ is diffeomorphic to $R^{n}$.

For a point $p \in M$; we set $d_{p}(x)=d(p, x)$. Notice that the distance function $d_{p}$ is not a smooth function (on the cut locus of $p$ ). Hence the critical points of $d_{p}$ are not defined in a usual sense. The notion of critical points of $d_{p}$ was introduced by Grove-Shiohama [GS].

A point $q(\neq p) \in M$ is called a critical point of $d_{p}$ if there is, for any non-zero vector $v \in T_{q} M$, a minimal geodesic $\gamma$ from $q$ to $p$ making an angle $\angle\left(v, \gamma^{\prime}(0)\right) \leq \frac{\pi}{2}$ with $v$. We simply say that $q$ is a critical point of $p$. It is now well-known that a complete noncompact Riemannian $n$-manifold $M$ is diffeomorphic to $R^{n}$ if there is a $p \in M$ such that $p$ has no critical points other than $p$.

Let $\Sigma$ be a closed subset of the unit tangent sphere $S_{p} M$ at $p \in M$. Let $B_{\Sigma}(p, r)$ denote the set of points $x \in B(p, r)$ such that there is a minimizing geodesic $\gamma$ from $p$ to $x$ with $\frac{d \gamma}{d t}(0) \in \Sigma$. For $0<r \leq \infty$, let $\Sigma_{p}(r)$ denote the set of unit vectors $v \in \Sigma$ such that the geodesic $\gamma(t)=\exp _{p}(t v)$ is minimizing on $[0, \mathrm{r})$. Notice that

$$
\Sigma_{p}\left(r_{2}\right) \subset \Sigma_{p}\left(r_{1}\right), 0<r_{1}<r_{2} ; \Sigma_{p}(\infty)=\bigcap_{r>0} \Sigma_{p}(r) .
$$

The following generalized Bishop-Gromov volume comparison theorem was observed in [S2].

Lemma 2.1. ([S2]) Let $(M, g)$ be a complete $n$-manifold with $\operatorname{Ric}_{M} \geq 0$. Let $\Sigma \subset S_{p} M$ be a closed subset. Then the function $r \rightarrow \frac{\operatorname{vol}\left[B_{\Sigma}(p, r)\right]}{\omega_{n} r^{n}}$ is monotone decreasing. 
Lemma 2.2. ([S2]) Let $(M, g)$ be a complete $n$-manifold with $\operatorname{Ric}_{M} \geq 0$. The function

$$
r \rightarrow \frac{\operatorname{vol}\left[B_{\Sigma_{p}(r)}(p, r)\right]}{\omega_{n} r^{n}}
$$

is monotone decreasing. If in addtion that $M$ has large volume growth, then

$$
\frac{\operatorname{vol}\left[B_{\Sigma_{p}(r)}(p, r)\right]}{\omega_{n} r^{n}} \geq \alpha_{M}, \quad \forall r>0 .
$$

Lemma 2.3. Let $(M, g)$ be a complete n-manifold with $\operatorname{Ric}_{M} \geq 0$ and $\alpha_{M}>0$. Then

$$
\frac{\operatorname{vol}\left[B_{\Sigma_{p}(\infty)}(p, r)\right]}{\omega_{n} r^{n}} \geq \alpha_{M}, \quad \forall r>0 .
$$

Proof. Observe that

$$
\frac{\operatorname{vol}\left[B_{\Sigma_{p}(r)}(p, r)\right]}{\omega_{n} r^{n}}=\frac{\operatorname{vol}\left[B_{\Sigma_{p}(\infty)}(p, r)\right]+\operatorname{vol}\left[B_{\Sigma_{p}(r) \backslash \Sigma_{p}(\infty)}(p, r)\right]}{\omega_{n} r^{n}}
$$

By the standard argument, we have

$$
\operatorname{vol}\left[B_{\Sigma_{p}(r) \backslash \Sigma_{p}(\infty)}(p, r)\right] \leq \frac{r^{n}}{n} \cdot \operatorname{vol}\left(\Sigma_{p}(r) \backslash \Sigma_{p}(\infty)\right)
$$

It follows from (2.2) that

$$
\lim _{r \rightarrow \infty} \operatorname{vol}\left(\Sigma_{p}(r) \backslash \Sigma_{p}(\infty)\right)=0 .
$$

Substituting (2.6) into (2.5) and letting $r \rightarrow \infty$, one obtains by virtue of (2.7) and $(2.3)$

$$
\begin{aligned}
\lim _{r \rightarrow \infty} \frac{\operatorname{vol}\left[B_{\Sigma_{p}(\infty)}(p, r)\right]}{\omega_{n} r^{n}} & \geq \lim _{r \rightarrow \infty} \frac{\operatorname{Vol}\left[B_{\Sigma_{p}(r)}(p, r)\right]}{\omega_{n} r^{n}} \\
& \geq \alpha_{M} .
\end{aligned}
$$

Using Lemma 2.1, one obtains (2.4).

Lemma 2.4. Let $(M, g)$ be a complete $n$-manifold with $\operatorname{Ric}_{M} \geq 0$ and $\alpha_{M}>0$. Let $R_{p}$ denote the (point set) union of rays issuing from $p$. Then for any $r>0$ and any $x \in \partial B(p, r)$,

$$
d\left(x, R_{p}\right) \leq 2 \alpha_{M}^{-\frac{1}{n}}\left\{\frac{\operatorname{vol}[B(p, r)]}{\omega_{n} r^{n}}-\alpha_{M}\right\}^{\frac{1}{n}} r .
$$


Proof. Let $s=d\left(x, R_{p}\right)$; then $s \leq r$ and

$$
B(x, s) \cup B_{\Sigma_{p}(\infty)}(p, 2 r) \subset B(p, 2 r) .
$$

The left hand side of (2.10) is a disjoint union. By (1.1), we have

$$
\operatorname{vol}(B(x, s)) \geq \alpha_{M} \omega_{n} s^{n} .
$$

From Lemma 2.1 and Lemma (2.3), one obtains

$$
\begin{aligned}
2^{n} \operatorname{vol}[B(p, r)] & \geq \operatorname{vol}[B(p, 2 r)] \\
& \geq \operatorname{vol}[B(x, s)]+\operatorname{vol}\left[B_{\Sigma_{p}(\infty)}(p, 2 r)\right] \\
& \geq \alpha_{M} \omega_{n} s^{n}+\alpha_{M} \omega_{n}(2 r)^{n} .
\end{aligned}
$$

thus

$$
s^{n} \leq 2^{n} r^{n} \alpha_{M}^{-1}\left\{\frac{\operatorname{vol}[B(p, r)]}{\omega_{n} r^{n}}-\alpha_{M}\right\} .
$$

This proves (2.9).

Let $p, q \in M$. The excess function $e_{p q}(x)$ is defined by

$$
e_{p q}(x):=d(p, x)+d(q, x)-d(p, q)
$$

Lemma 2.5. ([AG, S1]) Let $(M, g)$ be a complete $n$-manifold with $\operatorname{Ric}_{M}^{(k)} \geq 0$ for some $1 \leq k \leq n-1$. Let $\gamma:[0, a] \rightarrow M$ be a minimal geodesic from $p$ to $q$. Then for any $x \in M$,

$$
e_{p q}(x) \leq 8\left(\frac{s^{k+1}}{r}\right)^{\frac{1}{k}},
$$

where $s=d(x, \gamma), r=\min (d(p, x), d(q, x))$.

Let $\gamma:[0, \infty) \rightarrow M$ be a ray issuing from $p$ and let $x \in M$. It is easy to see that $e_{p, \gamma(t)}(x)=d(p, x)+d(\gamma(t), x)-t$ is decreasing in $t$ and that $e_{p, \gamma(t)}(x) \geq 0$. We define the excess function $e_{p, \gamma}$ associated to $p$ and $\gamma$ as

$$
e_{p, \gamma}(x)=\lim _{t \rightarrow+\infty} e_{p, \gamma(t)}(x) .
$$

Then

$$
e_{p, \gamma}(x) \leq e_{p, \gamma(t)}(x), \quad \forall t>0 .
$$

Lemma 2.6. Let $(M, g)$ be a complete open Riemannian manifold with $K_{p}^{\text {min }} \geq$ $-C$ for some $C>0$ and $p \in M$. Suppose that $x \neq p$ is a critical point of $p$. Then for any ray $\gamma:[0, \infty) \rightarrow M$ issuing from $p$

$$
e_{p, \gamma}(x) \geq \frac{1}{\sqrt{C}} \log \left(\frac{2}{1+e^{-2 \sqrt{C} d(p, x)}}\right) .
$$


Proof. For any $t>0$, take a minimal geodesic $\sigma_{t}:[0, d(x, \gamma(t))] \rightarrow M$ from $x$ to $\gamma(t)$. Since $x$ is a critical point of $p$, there exists a minimal geodesic $\tau$ from $x$ to $p$ such that $\sigma_{t}^{\prime}(0)$ and $\tau^{\prime}(0)$ make an angle at most $\frac{\pi}{2}$. Applying Lemma 1.1 to the geodesic triangle $\left(\left.\gamma\right|_{[0, t]}, \sigma_{t}, \tau\right)$, we obtain

$$
\cosh (\sqrt{C} t) \leq \cosh (\sqrt{C} d(x, \gamma(t))) \cosh (\sqrt{C} d(p, x)) .
$$

Multiplying the above inequality by $2 \exp (\sqrt{C}(d(p, x)-t))$ and letting $t \rightarrow+\infty$, we obtain

$$
\exp (\sqrt{C} d(p, x)) \leq \exp \left(\sqrt{C} e_{p, \gamma}(x)\right) \cosh (\sqrt{C} d(p, x)) .
$$

Then Lemma 2.6 follows from (2.17).

Proof of Theorem 2.1. We shall prove that $M$ contains no critical points of $p$ (other than $p$ ) and therefore it is diffeomorphic to $R^{n}$. To do this, take an arbitrary point $x(\neq p) \in M$ and set $r=d(p, x)$. It follows from (2.1) and (2.9) that

$$
d\left(x, R_{p}\right)<\left(\frac{1}{8 \sqrt{C}} \log \left(\frac{2}{1+e^{-2 \sqrt{C} r}}\right)\right)^{\frac{k}{k+1}} \cdot r^{\frac{1}{k+1}} .
$$

Thus we can find a ray $\gamma:[0,+\infty) \rightarrow M$ issuing from $p$ and satisfying

$$
s:=d(x, \gamma)<\left(\frac{1}{8 \sqrt{C}} \log \left(\frac{2}{1+e^{-2 \sqrt{C} r}}\right)\right)^{\frac{k}{k+1}} \cdot r^{\frac{1}{k+1}} .
$$

Take $q \in \gamma$ such that $d(x, q)=d(x, \gamma)$. By $(2.18), d(x, q)<r$. Also one can easily deduce from triangle inequality that

$$
\min (d(p, x), d(\gamma(t), x))=r, \quad \forall t \geq 2 r .
$$

Thus $q \in \gamma((0,2 r))$ and so

$$
d\left(x,\left.\gamma\right|_{[0,2 r]}\right)=s
$$

Using (2.12), (2.14) and (2.18), we obtain

$$
\begin{aligned}
e_{p, \gamma}(x) & \leq e_{p, \gamma(2 r)}(x) \\
& \leq 8\left(\frac{s^{k+1}}{r}\right)^{\frac{1}{k}} \\
& <\frac{1}{\sqrt{C}} \log \left(\frac{2}{1+e^{-2 \sqrt{C} r}}\right) .
\end{aligned}
$$


By (2.15) and (2.19), $x$ is not a critical point of $p$. Thus $M$ is diffeomorphic to $R^{n}$. This completes the proof of Theorem 2.1 .

Theorem 1.2 is a consequence of the following more general result.

Theorem 2.2. Let $(M, g)$ be a complete Riemannian $n$-manifold with $\operatorname{Ric}_{M}^{(k)} \geq$ $0, \alpha_{M}>0$. Suppose that $K_{p}^{\text {min }} \geq-C$ for some $p \in M$ and $C>0$. If

$$
\limsup _{r \rightarrow+\infty}\left\{\left(\frac{\operatorname{vol}[B(p, r)]}{\omega_{n} r^{n}}-\alpha_{M}\right) r^{\frac{k n}{k+1}}\right\}<2^{-n}\left(\frac{\log 2}{8 \sqrt{C}}\right)^{\frac{k n}{k+1}} \cdot \alpha_{M}
$$

then $M$ has finite topological type.

Proof of Theorem 2.2. By the Isotopy Lemma [C, G, GS], it suffices to show that for any $x \in M$, if $d(p, x)$ is large enough then $x$ is not a critical point of $p$. Our assumption (2.20) enables us to find a small number $\epsilon>0$ and a sufficiently large $r_{1}$ such that

$$
\left(\frac{\operatorname{vol}[B(p, r)]}{\omega_{n} r^{n}}-\alpha_{M}\right) r^{\frac{k n}{k+1}}<2^{-n}\left(\frac{\log 2}{8 \sqrt{C}}-\epsilon\right)^{\frac{k n}{k+1}} \alpha_{M}, \quad \forall r \geq r_{1}
$$

Since

$$
\lim _{r \rightarrow+\infty} \log \left(\frac{2}{1+e^{-2 \sqrt{C} r}}\right)=\log 2,
$$

there is a sufficiently large $r_{2}$ such that

$$
\frac{\log \left(\frac{2}{1+e^{-2 \sqrt{C} r}}\right)}{8 \sqrt{C}}>\frac{\log 2}{8 \sqrt{C}}-\epsilon, \quad \forall r \geq r_{2} .
$$

Let $r_{0}=\max \left(r_{1}, r_{2}\right)$; then for any $r \geq r_{0}$ we have from $(2.21)$ and $(2.22)$ that

$$
\begin{aligned}
\frac{\operatorname{vol}[B(p, r)]}{\omega_{n} r^{n}} & <\left\{1+2^{-n}\left(\frac{\frac{\log 2}{8 \sqrt{C}}-\epsilon}{r}\right)^{\frac{k n}{k+1}}\right\} \cdot \alpha_{M} \\
& <\left\{1+2^{-n}\left(\frac{1}{8 \sqrt{C} r} \log \left(\frac{2}{1+e^{-2 \sqrt{C} r}}\right)\right)^{\frac{k n}{k+1}}\right\} \cdot \alpha_{M}
\end{aligned}
$$

Now one can repeat the arguments as in the proof of Theorem 2.1 to prove that $M \backslash B\left(p, r_{0}\right)$ contains no critical points of $p$. Therefore $M$ has finite topological type. This completes the proof of Theorem 2.2. 
Proof of Theorem 1.3. Let $\delta=\delta(C)<\frac{1}{20}$ be a solution of the following inequality

$$
\cosh ^{2}(4 \sqrt{C} \delta)-\cosh (6 \sqrt{C} \delta)<0 .
$$

We take our $\epsilon=\epsilon(n, C)$ in Theorem 1.3 to be

$$
\epsilon=\left(\frac{\delta}{8}\right)^{n}
$$

Take an arbityary point $x(\neq p) \in M$ and let $r=d(p, x)$. It suffices to prove that $x$ is not a critical point of $p$. Let $\gamma:[0,2 r] \rightarrow M$ be a minimizing geodesic from $p$ to $q=\gamma(2 r)$ such that $s:=d(x, \gamma)=d\left(x, B_{\Sigma_{p}(\infty)}(p, 2 r)\right)$. Using the same arguments as in the proof of (2.9), we obtain

$$
d\left(x, B_{\Sigma_{p}(\infty)}(p, 2 r)\right) \leq 2 \alpha_{M}^{-\frac{1}{n}}\left\{\frac{\operatorname{vol}[B(p, r)]}{\omega_{n} r^{n}}-\alpha_{M}\right\}^{\frac{1}{n}} \cdot r .
$$

Take a minimizing geodesic $\sigma$ from $x$ to $q$. For any minimal geodesic $\sigma_{1}$ from $x$ to $p$, let $\tilde{p}=\sigma_{1}(\delta r)$ and $\tilde{q}=\sigma(\delta r)$. Applying the Toponogov comparison theorem to the hinge $\left(\left.\sigma\right|_{[0, \delta r]},\left.\sigma_{1}\right|_{[0, \delta r]}\right)$ in $M-B_{\frac{r}{4}}(p)$, we have

$$
\cosh \left(\frac{4 \sqrt{C}}{r(x)} d(\tilde{p}, \tilde{q})\right) \leq \cosh ^{2}(4 \sqrt{C} \delta)-\sinh ^{2}(4 \sqrt{C} \delta) \cos \theta
$$

where $\theta=\angle\left(\sigma^{\prime}(0), \sigma_{1}^{\prime}(0)\right)$ be the angle of $\sigma$ and $\sigma_{1}$ at $x$ and we have used the fact that the sectional curvature of $M$ satisfies $K_{M} \geq-\frac{4^{2} C}{r^{2}}$ on $M-B_{\frac{r}{4}}(p)$. Let $m \in \gamma$ such that $d(x, m)=d(x, \gamma)$; it then follows from the triangle inequality that

$$
\begin{aligned}
d(\tilde{p}, \tilde{q}) \geq & d(p, q)-d(p, \tilde{p})-d(q, \tilde{q}) \\
= & d(p, m)+d(q, m)-[d(p, x)-d(\tilde{p}, x)] \\
& \quad-[d(x, q)-d(x, \tilde{q})] \\
= & 2 \delta r+[d(p, m)-d(p, x)]+[d(q, m)-d(q, x)] \\
\geq & 2 \delta r-2 d(x, m) .
\end{aligned}
$$

From (2.25), (2.26) and our assumption (1.5), we have

$$
\begin{aligned}
d(x, m) & =d\left(x, B_{\Sigma_{p}(\infty)}(p, 2 r)\right) \\
& \leq 2 \epsilon^{\frac{1}{n}} r \\
& \leq \frac{\delta r}{4} .
\end{aligned}
$$


Thus we have

$$
d(\tilde{p}, \tilde{q}) \geq \frac{3}{2} \delta r
$$

Substituting (2.30) into (2.27) and using (2.24), we find that

$$
\begin{aligned}
\sinh ^{2}(4 \sqrt{C} \delta) \cos \theta & \leq \cosh ^{2}(4 \sqrt{C} \delta)-\cosh \left(\frac{4 \sqrt{C}}{r(x)} d(\tilde{p}, \tilde{q})\right) \\
& \left.\leq \cosh ^{2}(4 \sqrt{C} \delta)-\cosh (6 \sqrt{C} \delta)\right) \\
& <0,
\end{aligned}
$$

or

$$
\theta>\frac{\pi}{2}
$$

Hence $x$ is not a critical point of $p$. Thus $M$ is diffoemorphic to $R^{n}$. The theorem follows.

\section{References}

[A] U. Abresh, Lower curvature bounds, Toponogov's Theorem and bounded topology I, Ann. Sci. École Norm. Sup. 18 (1985), 651-670.

[AG] U. Abresch, D. Gromoll, On complete manifolds with nonnegative Ricci curvature, J. Amer. Math. Soc. 3 (1990), 355-374.

[An] M. Anderson, On the topology of complete manifolds of nonegative Ricci curvature, Topology 29 (1990) 41-55.

[BC] R. L. Bishop, R. J. Crittenden, Geometry of manifolds, Academic Press, New York 1964.

[CX] M. do Carmo, C. Y. Xia, Ricci curvature and the topology of open manifolds, preprint.

[C] J. Cheeger, Critical points of distance functions and applications to geometry, Lecture notes in Math., vol. 1504, Springer-Verlag, New York 1991, pp. 1-38.

[CC] J. Cheeger, T. Colding, On the structure of spaces with Ricci curvature bounded from below, J. Diff. Geom. 46 (1997), 406-480.

[G] M. Gromov, Curvature, diameter and Betti numbers, Comment. Math. Helv. 56 (1981), 179-195.

[GLP] M. Gromov, J. Lafontaine, P. Pansu, Structures métrique pour les variétes Riemanniennes. Cédic/Fernand, Nathan, Paris 1981.

[GS] K. Grove, K. Shiohama, A generalized sphere theorem, Ann. Math. 106 (1977), 201-211.

[L] P. Li, Large time behavior of the heat equation on complete manifolds with nonnegative Ricci curvature, Ann. of Math. 124 (1986), 1-21.

[M1] Y. Machigashira, Manifolds with pinched radial curvature,Proc. Amer. Math. Soc. 118 (1993), 979-985.

[M2] Y. Machigashira, Complete open manifolds of nonnegative radial curvature, Pacific J. Math. 165 (1994), 153-160.

[P] G. Perelman, Manifolds of positive Ricci curvature with almost maximal volume, J. Amer. Math. Soc. 7 (1994), 299-305.

$[\mathrm{Pe}]$ P. Petersen, Comparison geometry problem list, Riemannian geometry (Waterloo, ON, 1993), 87-115, Fields Inst. Monogr., 4, Amer. Math. Soc., Providence, RI 1996.

[SS] J. Sha and Z. Shen, Complete manifolds with nonnegative Ricci curvature and quadratically nonnegatively curved infinity, Amer. J. Math. 119 (1997), 1399-1404. 
[S1] Z. Shen, On complete manifolds of nonnegative $k$ th-Ricci curvature, Trans. Amer. Math. Soc. 338 (1993), 289-310.

[S2] Z. Shen, Complete mnifolds with nonnegative Ricci curvature and large volume growth, Invent. Math. 125 (1996), 393-404.

Changyu Xia

Departamento de Matemática-IE

Universidade de Brasília

Campus Universitário

70910-900-Brasília-DF

Brasil

e-mail: xia@ipe.mat.unb.br

(Received: August 17, 1998) 